European
Neurology

Eur Neurol 2010;64:236-240

DOI: $\underline{10.1159 / 000317583}$
Received: April 16, 2010

Accepted: June 22, 2010

Published online: September 4, 2010

\title{
Natalizumab and Beyond
}

\author{
Murat Yildiz Barbara Tettenborn Norman Putzki \\ Department of Neurology, Cantonal Hospital St. Gallen, St. Gallen, Switzerland
}

Dear Sir,

Natalizumab is the first monoclonal antibody licensed for the treatment of relapsing-remitting multiple sclerosis (RRMS) [1]. Natalizumab was licensed by the European Medicines Agency in 2006 with a restricted indication [2]. Mainly, natalizumab is used for patients with insufficient response to alternative first-line disease-modifying therapies (DMTs), i.e. $\beta$-interferon (IFN- $\beta$ ) or glatiramer acetate (GA), or for patients with aggressive MS. Intolerance of other DMTs may occasionally also account for switching to natalizumab.

No prospective, randomized placebocontrolled trials have been conducted to provide class I or II evidence-based guidance for treatment decisions in patients with insufficient response to first-line DMTs. Also, it is unclear how to treat patients who discontinue natalizumab treatment. However, discontinuation occurs in approximately $10 \%$ in clinical practice due to hypersensitivity, neutralizing antibodies (NABs) against natalizumab, insufficient response or a combination of these [3]. Considering that about 60,000 patients worldwide have had exposure to natalizumab (of whom 6,000 will not continue this treatment) [4], it is of clinical relevance to investigate strategies for patients who were eligible for natalizumab treatment but who have to discontinue it.

The following case series aims (i) to exemplify clinically relevant reasons for dis- continuation of natalizumab treatment and (ii) to illustrate the spectrum of alternative treatment strategies when natalizumab is no longer an option.

\section{Case 1}

This 52-year-old female had been suffering from RRMS since 2004.

DMT was initiated with subcutaneous IFN- $\beta_{1 \mathrm{a}}$ in late 2006.

Her annual relapse rate (ARR) on IFN$\beta$ at that time was 1. In December 2007, she experienced a severe relapse caused by myelitis of the dorsal spine despite DMT with IFN- $\beta$. The Expanded Disability Status Scale (EDSS) increased from 2.0 to 3.5 with the last relapse.

On MRI, 1 new paraventricular right frontal lesion and 1 preexisting lesion with gadolinium $(\mathrm{Gd})$ enhancement in the medulla were found beside acute myelitis.

Relapse treatment consisted of 2 pulses with intravenous methylprednisolone (1 g for 5 consecutive days, $2 \mathrm{~g}$ for another 5 days) which led to partial remission (EDSS 3.0).

Treatment with IFN- $\beta$ was stopped, and natalizumab was introduced (Tysabri $^{\circledR}, 300$ mg i.v. every 4 weeks; Biogen Idec, Cambridge, Mass., USA) after a 2-week DMT-free interval. She remained relapse free for 8 months before she was hospitalized with right-sided hypo-acusis, severe

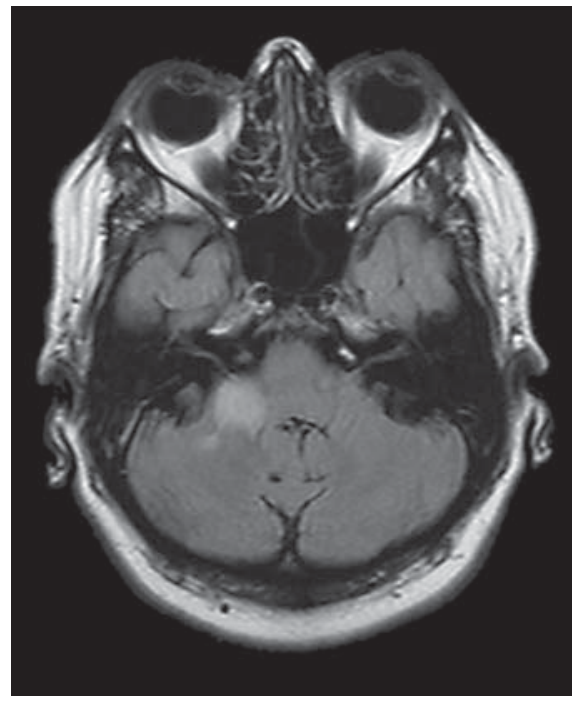

Fig. 1. MRI: 1.5-tesla $T_{2}$-weighted image. The patient presented with a severe relapse during natalizumab treatment, and symptoms corresponded to this large new lesion in the cerebellum.

ataxia and vertigo. An MRI revealed 5 new cerebral lesions - the largest one in the right cerebellar peduncle was responsible for her symptoms (fig. 1).

Natalizumab was temporarily withdrawn to further investigate the case. CSF investigation excluded JC virus infection,

\section{KARGER}

Fax +4161306 1234 E-Mail karger@karger.ch www.karger.com
(C) 2010 S. Karger AG, Basel 0014-3022/10/0644-0236\$26.00/0

Accessible online at:

www.karger.com/ene
Norman Putzki, MD

Department of Neurology, Multiple Sclerosis Outpatient Clinic

Cantonal Hospital St. Gallen

Rorschacher Strasse 95, CH-9007 St. Gallen (Switzerland)

Tel. +41 71494 1669, Fax +41 71495 2895, E-Mail norman.putzki@ kssg.ch 
and pulsed steroid treatment led to incomplete remission. Within the following month, she experienced another episode with double vision and hypaesthesia of the right face which responded favourably to steroids. At that time she was persistently positive for NAB against natalizumab (2 tests 2 months apart), and treatment was terminated. After a 3-month 'wash-out' period, off-label therapy with rituximab (RX) was initiated (2 g i.v. every 6 months). She has been relapse and progression free for $>12$ months; MRI did not show any further disease activity.

\section{Case 2}

This 39-year-old male patient had been known for RRMS since 2003 and long-standing, clinically silent primary biliary cirrhosis. He experienced a relapse in late 2004 (EDSS temporarily increased to 4.0) and subcutaneous IFN- $\beta_{1 \mathrm{a}}$ was initiated. After 2 injections with IFN- $\beta_{1 \mathrm{a}} 22$ $\mu g$ s.c., upper abdominal pain occurred and suggested hepatic problems. IFN- $\beta$ treatment had to be stopped due to liver enzyme elevation exceeding 5 times the upper limits of normal (liver enzymes were normal before IFN- $\beta$ initiation). Treatment with GA was started but severe skin reactions led to early discontinuation. Subsequently, he was left untreated for 2 years and he remained relapse free. A follow-up MRI 2 years later showed 9 new $\mathrm{T}_{2}$-weighted lesions (compared to 2006, $20 \mathrm{~T}_{2}$-weighted lesions in total) without Gd enhancement; EDSS was 1.0. At that time, natalizumab was licensed, and treatment was initiated due to intolerance of IFN- $\beta / \mathrm{GA}$ and subclinical progression.

After 1 year of natalizumab treatment, persistent NABs were found in 2 routine controls 3 months apart. Therefore, natalizumab was permanently discontinued in April 2009. MRI showed no new lesions and no Gd enhancement but partial remission of previous lesions. Intramuscular IFN- $\beta_{1 \mathrm{a}}$ once weekly was introduced in July 2009 with slow dose titration and frequent laboratory controls. IFN- $\beta$ treatment was well tolerated without liver enzyme elevation. The patient remained relapse free for a further 6 months until he stopped IFN- $\beta$ because of unwillingness to continue injection therapy.

\section{Case 3}

This 32-year-old female patient was diagnosed as having RRMS in 2004. DMT with IFN $-\beta_{1 \mathrm{a}} 22 \mu \mathrm{g}$ s.c. $(3 \times$ per week) was initiated in October 2004 two months after a relapse (EDSS 3.0, decreased to EDSS 2.0 after steroid treatment). During 2 years, the ARR was 1 on subcutaneous IFN- $\beta_{1 a}$ treatment. Afterwards, she experienced 2 relapses on IFN- $\beta_{\text {la }} 44 \mu \mathrm{g}$ s.c. within 2007 (EDSS 3.0). She was compliant. MRI showed a total of $10 \mathrm{~T}_{2}$-weighted lesions (slight progression over 2 years) and 1 new Gd-enhanced lesion in the cervical myelon. Natalizumab was started in February 2008 and was well tolerated (EDSS 2.0). In June 2009, she had a relapse with signs of cervical myelitis. MRI showed 1 new Gd-enhanced lesion in the left superior frontal gyrus (compared to 2007) and cervical myelitis. After pulsed steroid therapy, symptoms fully remitted (EDSS 2.0). Natalizumab therapy was permanently discontinued in September 2009 due to persistent NAB. EDSS was 2.0 at cessation of natalizumab therapy. A follow-up MRI showed 1 new $\mathrm{T}_{2}$-weighted lesion with contrast enhancement. DMT with GA was initiated in January 2009. She has been relapse free with GA for $>12$ months (last EDSS 2.0).

\section{Case 4}

This 49-year-old female patient was diagnosed as having RRMS in 2003. She was treated with intramuscular IFN- $\beta_{1 a}$ once per week from November 2003 until November 2005. Due to an ARR of 2 on intramuscular IFN- $\beta_{1 a}$, treatment was switched to IFN- $\beta_{1 \mathrm{a}} 44 \mu \mathrm{g}$ s.c. $(3 \times$ per week $)$. The status of NABs against IFN- $\beta$ was not investigated. The ARR decreased to 1.0 before she had a severe relapse with paresis of the right arm and only partial response to steroid therapy. Her EDSS increased from 0 to 4.5 from 2003 to 2007 . When treatment with natalizumab was initiated in August 2007, she experienced a severe hypersensitivity reaction during the second infusion, and natalizumab was discontinued. Later, she was also found persistently NAB positive.

From January to May 2008, we opted for mitoxantrone (MX) induction therapy $\left(12 \mathrm{mg} / \mathrm{m}^{2}\right.$ body surface monthly for $3 \mathrm{cy}$ cles). In June 2008, MX was continued with $9 \mathrm{mg} / \mathrm{m}^{2}$ body surface, and administration every 3 months in combination with GA was planned. While she had been relapse free during induction therapy with MX, a relapse occurred afterwards (EDSS 4.5). Pulsed steroid therapy was applied, and $\mathrm{MX}$ at $9 \mathrm{mg} / \mathrm{m}^{2}$ was introduced. At the end of 2008, GA monotherapy was attempted. Two further relapses occurred during 2009 which resulted in a temporary worsening of gait. Follow-up MRIs between August 2007 and recently did not show any new lesions or Gd enhancement (EDSS 4). Re-introduction of MX was scheduled.

\section{Case 5}

This 18-year-old female student was diagnosed as having RRMS at the age of 15 (in 2006). She suffered from aggressive MS with 4 relapses in 2006. Despite introduction of DMT with subcutaneous IFN- $\beta_{1 a}$, she experienced 2 additional relapses over the next 9 months (until mid 2007). Already 14 months after the diagnosis of MS, she had had 6 relapses and progression to EDSS 3.5 with the last relapse. MRI demonstrated $3 \mathrm{~T}_{2}$-weighted lesions in the brain and 2 additional lesions in the cervical spine, one of which showed Gd enhancement. Although not licensed for individuals $<18$ years of age, it was decided to initiate natalizumab treatment. Two days after the second infusion of natalizumab, she developed a skin rash which was interpreted as delayed hypersensitivity to natalizumab and treatment was discontinued (end of 2007). Neither laboratory tests nor NAB tests were undertaken at that time since her general practitioner did not assume a relationship between the rash and natalizumab treatment. It was decided to initiate MX treatment after a 3-month 'wash-out' period. During that time she experienced a prolonged relapse with ataxia and signs of myelitis. Subsequently, MX was started in April 2008 (monthly treatment with $10 \mathrm{mg} / \mathrm{m}^{2}$ body surface for 4 months). Two weeks after the first dose of MX, she had a relapse with cervical myelitis. Further infusions of MX were combined with a pulsed steroid therapy for 3 infusions. MX was continued until May 2009 with a total 7 cycles of MX and a cumulative dose of $85 \mathrm{mg} / \mathrm{m}^{2}$. In September 2008, she also started GA which was well tolerated. Compliance to GA was unsatisfactory with only 3 injections per week. For 17 months, no further relapses occurred. Relapse activity remit- 
ted in October 2009 and December 2009, on both occasions with signs of cervical and dorsal myelitis. MRI of the brain demonstrated remission of previous lesions and no contrast enhancement while MRI of the spine showed progression of a preexisting lesion in the cervical spine. GA was terminated in December 2009. After laboratory tests failed to demonstrate NABs against natalizumab, re-exposure to natalizumab (preceded by pretreatment with low-dose steroids and antihistamines) did not lead to hypersensitivity, and treatment will be continued.

\section{Discussion}

The patients presented here mostly exhibited substantial clinical and MRI disease activity despite treatment with DMT prior to treatment initiation with natalizumab. When therapy with first-line DMT is not successful, the patient should be assessed to identify possible reasons (e.g. non-adherence, NABs to IFN- $\beta$, infections or even the wrong diagnosis). For patients with worsening RRMS, other therapeutic options, including natalizumab, immunosuppression with MX (or cyclophosphamide) and scheduled add-on pulse therapy with corticosteroids, are available $[5,6]$, but the evidence level for any of these approaches is low. It is unknown whether the risk-benefit profile of any of these treatment regimens offers advantages in comparison to therapy with natalizumab [7].

Monotherapy with natalizumab is mainly recommended for patients who had recent inflammatory disease activity and evidence that alternative DMTs have been ineffective. Such individuals appear to experience a significant reduction of clinical and MRI disease activity [8-10]. Nevertheless, about $10 \%$ of patients need to discontinue natalizumab in clinical practice [3, 11]. Failure of natalizumab treatment can be addressed by switching to other DMTs (GA, IFN- $\beta$, MX, RX) but little information regarding the effectiveness of such strategies is available.

In the cases presented here, natalizumab treatment was suspended because of disease activity due to occurrence of NABs against natalizumab (cases 1 and 3) and/or hypersensitivity reaction associated with therapy (cases 4 and 5) and on the basis of persistent NAB against natalizumab without clinical disease activity or hypersensitivity (case 2).
It is questionable if re-introduction of the previous class of DMT (GA vs. IFN- $\beta$ ) can be a sensible option. Testing for NAB against IFN- $\beta$ can be of help in this decision-making process, especially when breakthrough disease occurred during previous IFN- $\beta$ treatment. There is accumulating evidence that persistent high titres of NABs against IFN- $\beta$ reduce the biological activity of IFN- $\beta$ which is sooner or later associated with an increase in disease activity [12]. Thus, the European Neurological Society recommended routine NAB testing [13]. A previous study observed that patients who were switched from IFN- $\beta$ to GA or MX had a better response than those switched to another IFN- $\beta$ preparation [14]. A less favourable outcome after switching between IFN- $\beta$ preparations was also found earlier [15]. Furthermore, switching from IFN- $\beta$ to GA because of persistent clinical disease activity led to reduction of the ARR by $61 \%$ [16] (all studies class III-IV evidence). In case of high disease activity, a combination of GA and MX [17] may also be taken into consideration (cases 4 and 5). One study found that MX-GA combination therapy resulted in a $70 \%$ reduction of Gd-enhanced lesions after a year versus GA alone. Mean ARRs were 0.16 and 0.32 in the MX-GA and GA groups, respectively [18].

Also MX monotherapy was shown to be effective ( 5 and $12 \mathrm{mg} / \mathrm{m}^{2}$ ) given every 3 months over 2 years [19], also in aggressive RRMS (monthly MX for 6 months followed by maintenance treatment) [20]. To date, MX is the only immunosuppressant approved for MS, but there are concerns about the safety of MX. Potentially lifethreatening congestive heart failure was reported in up to $6.0 \%$ of patients with solid tumours or leukaemia receiving MX [21, 22] but rates in monotherapy for MS may be lower [23]. An asymptomatic left ventricular ejection fraction of $<50 \%$ was 3 times higher with a cumulative dose of $>100 \mathrm{mg} / \mathrm{m}^{2}(5.0 \%)$ than below [23]. The risk for cardiomyopathy limits the approved cumulative dose of MX to a maximum of $140 \mathrm{mg} / \mathrm{m}^{2}$ [24].

A second major concern is the development of acute myeloid leukaemia (AML). In an older review of 1,378 medical records of MS patients treated with MX, the incidence of AML was $0.07 \%$ [25]. Recently, a new study from Italy raised concerns since the rate of AML was reported to be 10 times higher. Leukaemia arose after an av- erage of 3 years after the first use of the drug and 18 months after MX treatment had been terminated. Again, the risk increase was dose dependent [26]. In another study, the majority of AML cases occurred in patients exposed to $>60 \mathrm{mg} / \mathrm{m}^{2}$ [27].

Gender and age of the patients are other important factors to be considered. In women less than 35 years of age, prolonged amenorrhoea has been described in approximately $7 \%$, and fertility may also be permanently impaired [28].

Cyclophosphamide is used as an alternative immunosuppressant in some countries but adequate placebo-controlled trials comparable to the pivotal studies for DMT have never been conducted and the drug is not licensed for MS. Patients with aggressive RRMS may profit more from cyclophosphamide than patients with progressive disease [29]. Aside from acute toxicity, main concerns with cyclophosphamide refer to secondary malignancy [30].

RX seems to be a promising off-label alternative in individual cases. RX is an antibody directed against the CD20 antigen on $\mathrm{B}$ cells and leads to long-standing depletion of B cells and pre-B cells. Currently it is approved for treatment of B-cell lymphoma and rheumatoid arthritis. RX has initially been investigated in neuromyelitis optica where treatment resulted in a reduction of relapses and improvement of disability [31]. Also in MS, B-cell pathology is being increasingly recognized as important [32]. A single course of RX largely reduced inflammatory brain lesions and clinical relapses for 48 weeks in RRMS. RX use was associated with rare cases of progressive multifocal leuko-encephalopathy, further side effects include an increased risk of (opportunistic) infections. In case 1, RX was used for the treatment of aggressive RRMS, which led to stabilization of disease activity. RX was favoured over MX based on personal experience that currently extends to a further 10 RRMS patients in similar conditions. Similarly, 2 further cases where standard therapy had failed were reported to become stable with long-term therapy with RX [33]. Lack of reimbursement may limit the off-label use of RX.

In case 5, natalizumab was re-introduced despite the fact that the patient had previously developed a delayed hypersensitivity-like reaction to natalizumab. Treatment with MX appeared successful in her case; however, de-escalation to GA was not. We did not suggest continuous 
MX treatment for safety considerations. Any sort of hypersensitivity (regardless of NAB findings) represents a contra-indication to natalizumab, and further exposure may put patients at risk of serious hypersensitivity reactions. It is recommended to investigate $\mathrm{NAB}$ against natalizumab on a routine basis [34] (e.g. after 6 months of natalizumab treatment), and it is especially warranted when hypersensitivity-like reactions occur. Hellwig et al. [35] described 2 patients with delayed systemic reactions beginning several hours to days after the first infusion. But as neither of the patients tested was positive for NABs, natalizumab therapy was continued and reactions decreased over time [35].
In summary, all treatment decisions were made on an individual basis in an environment of scientific uncertainty. While these cases shall not encourage the use of one or the other strategy, they are meant to illustrate the wide and increasingly complex spectrum of DMT in RRMS. In practice, even more treatment approaches are available (e.g. alemtuzumab, daclizumab, intravenous cladribine, just to name a few). Treatment decisions for patients in this case series have not been exclusively made at our centre. Most patients were referred after insufficient control with first-line DMTs or when complications with natalizumab occurred. Although guidelines for adequate monitoring of RRMS have been suggested [36], there is an ongoing debate how to define 'non-response' and how this can be addressed. The urgent need for placebo-controlled trials for patients with insufficient response is apparent.

\section{Conflicts of Interest}

N.P. has received honoraria, travel grants, research grants and personal compensation from Bayer Healthcare, Biogen Idec, GSK, TEVA, Sanofi Aventis, Merck Serono and Novartis. B.T. has received travel grants and honoraria from Merck Serono, Biogen Idec and Bayer Healthcare.

\section{References}

1 Polman CH, O’Connor PW, Havrdova E, Hutchinson M, Kappos L, Miller DH, et al: A randomized, placebo-controlled trial of natalizumab for relapsing multiple sclerosis. $\mathrm{N}$ Engl J Med 2006;354:899-910.

2 European Medicines Agency. Natalizumab Elan Pharma: natalizumab.http://www.ema. europa.eu/ema/index.jsp?curl=pages $/$ medicines/human/medicines/000624/human med_000919.jsp\&mid=WC0b01ac058001d 1 $24 \&$ murl=menus $/$ medicines $/$ medicines. jsp\&jsenabled=true.

-3 Putzki N, Yaldizli O, Bühler R, et al: Natalizumab reduces clinical and MRI activity in multiple sclerosis patients with high disease activity: results from a multicenter study in Switzerland. Eur J Neurol 2010;63:101-106.

4 FDA: FDA Drug Safety Communication: Risk of Progressive Multifocal Leukoencephalopathy (PML) with the use of Tysabri (natalizumab). http://www.fda.gov/Drugs/ DrugSafety/PostmarketDrugSafetyInformationforPatientsand Providers/ucm 199872.htm (accessed February 8, 2010).

5 Confavreux C, Vukusic S: Non-specific immunosuppressants in the treatment of multiple sclerosis. Clin Neurol Neurosurg 2004; 106:263-269.

6 Rizvi SA, Agius MA: Current approved options for treating patients with multiple sclerosis. Neurology 2004;63(suppl 6):S8-S14

7 Dorsey ER, Thompson JP, Noyes K, et al: Quantifying the risks and benefits of natalizumab in relapsing multiple sclerosis. Neurology 2007;68:1524-1528.

-8 Sorensen PS: How effective is natalizumab as second-line treatment for multiple sclerosis in daily clinical praxis? Eur J Neurol 2009;16: 287-288.
9 Oturai AB, Koch-Henriksen N, Petersen T, Jensen PE, Sellebjerg F, Sorensen PS: Efficacy of natalizumab in multiple sclerosis patients with high disease activity: a Danish nationwide study. Eur J Neurol 2009;16:420-423.

10 Hutchinson M, Kappos L, Calabresi PA: The efficacy of natalizumab in patients with relapsing multiple sclerosis: subgroup analyses of AFFIRM and SENTINEL. J Neurol 2009; 256:405-415.

11 Putzki N, Kollia K, Woods S, et al: Natalizumab is effective as second-line therapy in the treatment of relapsing remitting MS. Eur J Neurol 2009;16:424-426.

12 Pachner AR, Warth JD, Pace A: Effect of neutralizing antibodies on biomarker responses to interferon beta: the INSIGHT study. Neurology 2009;73:1493-1500.

13 Hartung HP, Munschauer F, Schellekens H: Significance of neutralizing antibodies to interferon beta during treatment of multiple sclerosis: expert opinions based on the proceedings of an international consensus conference. Eur J Neurol 2005; 12:588-601.

14 Caon C, Din M, Ching W, et al: Clinical course after change of immunomodulating therapy in relapsing-remitting multiple sclerosis. Eur J Neurol 2006;13:471-474.

15 Limmroth V, Putzki N: High-dose, frequently administered interferon-beta. J Neurol Sci 2005; 15:95-96.

16 Carra A, Onaha P, Luetic B, et al: Therapeutic outcome 3 years after switching of immunomodulatory therapies in patients with relapsing-remitting multiple sclerosis in Argentina. Eur J Neurol 2008;15:386-393.

17 Gold R: Combination therapies in multiple sclerosis. J Neurol 2008;255(suppl 1):51-60.

18 Vollmer T, Panitch H, Bar-Or A, et al: Glatiramer acetate after induction therapy with mitoxantrone in relapsing multiple sclerosis. Mult Scler 2008;14:663-670.
9 Hartung HP, Gonsette R, König N, et al: Mitoxantrone in progressive multiple sclerosis: a placebo-controlled, double-blind, randomised, multicentre trial. Lancet 2002;360: 2018-2025.

20 Emmanuelle Le Page E, Leray E, Taurin G, et al: Mitoxantrone as induction treatment in aggressive relapsing remitting multiple sclerosis: treatment response factors in a 5 -year follow-up observational study of 100 consecutive patients. J Neurol Neurosurg Psychiatry 2008;79:52-56.

21 Goffette S, van Pesch V, Vanoverschelde JL, et al: Severe delayed heart failure in three multiple sclerosis patients previously treated with mitoxantrone. J Neurol 2005;252:12171222.

22 Rivera V, AL-Sabbagh A, Bennett R, et al: RENEW study update XVIII: ongoing evaluation of the safety and tolerability of mitoxantrone in worsening multiple sclerosis. Mult Scler 2008; 14:S29.

23 Ghalie RG, Edan G, Laurent M, et al: Cardiac adverse effects associated with mitoxantrone (Novantrone) therapy in patients with MS. Neurology 2002;59:909-913.

24 Cohen BA, Mikol DD: Mitoxantrone treatment of multiple sclerosis. Neurology 2004; 63(suppl 6):S28-S32.

25 Ghalie RG, Mauch E, Edan G, et al: A study of therapy-related acute leukaemia after mitoxantrone therapy for multiple sclerosis. Mult Scler 2002;8:441-445.

26 Martinelli L: Late-breaking science program at the American Academy of Neurology's 61st Annual Meeting in Seattle, April 25 to May 2, 2009. http://www.aan.com/press/index.cfm?fuseaction $=$ release.view\&release $=$ 725 (accessed February 8, 2010). 
27 Ellis R, Boggild M: Therapy-related acute leukaemia with mitoxantrone: what is the risk and can we minimise it? Mult Scler 2009; 15:505-508.

28 Edan G, Brochet B, Clanet M, et al: Safety profile of mitoxantrone in a cohort of 802 multiple sclerosis patients: a 4-year mean follow-up study. Neurology 2004;62(suppl 5):A493.

29 Weiner HL, Mackin GA, Orav EJ, et al: Intermittent cyclophosphamide pulse therapy in progressive multiple sclerosis: final report of the Northeast Cooperative Multiple Sclerosis Treatment Group. Neurology 1993;43: 910-1008.
30 Weiner HL, Cohen JA: Treatment of multiple sclerosis with cyclophosphamide: critical review of clinical and immunologic effects. Mult Scler 2002;8:142-154.

31 Cree BA, Lamb S, Morgan K, et al: An open label study of the effects of rituximab in neuromyelitis optica. Neurology 2005;64:12701272.

>32 Meinl E, Krumbholz M, Hohlfeld R: B lineage cells in the inflammatory central nervous system environment: migration, maintenance, local antibody production, and therapeutic modulation. Ann Neurol 2006; 59:880-892.

-33 Stüve O, Leussink VI, Fröhlich R, et al: Longterm B-lymphocyte depletion with rituximab in patients with relapsing-remitting multiple sclerosis. Arch Neurol 2009;66: 259-261.
34 Kappos L, Bates D, Hartung HP, et al: Natalizumab treatment for multiple sclerosis: recommendations for patient selection and monitoring. Lancet Neurol 2007;6:431-441.

35 Hellwig K, Schimrigk S, Fischer M, et al: Allergic and nonallergic infusion reactions during natalizumab therapy. Arch Neurol 2008;65:656-658.

36 Sellebjerg F, Barnes D, Filippini G, et al: EFNS guideline on treatment of multiple sclerosis relapses: report of an EFNS task force on treatment of multiple sclerosis relapses. Eur J Neurol 2005;12:939-946. 